Cell Research (1999), 9, 27-35

\title{
Foliar modifications induced by inhibition of polar transport of auxin
}

\author{
Ni Di An, Ling Jian WANG, Zhi Hong XU ${ }^{1}$, Zhen \\ Ao XIA \\ Shanghai Institute of Plant Physiology, Chinese Academy of \\ Sciences, Shanghai 200032, China
}

\begin{abstract}
The effects of auxin polar transport inhibitors, 9-hydroxyfluorene-9-carboxylic acid (HFCA); 2, 3, 5-triiodobenzoic acid (TIBA) and trans-cinnamic acid (CA) on leaf pattern formation were investigated with shoots formed from cultured leaf explants of tobacco and cultured pedicel explants of Orychophragmus violaceus, and the seedlings of tobacco and Brassica chinensis. Although the effective concentration varies with the inhibitors used, all of the inhibitors induced the formation of trumpet-shaped and/or fused leaves. The frequency of trumpet-shaped leaf formation was related to the concentration of inhibitors in the medium. Histological observation of tobacco seedlings showed that there was only one main vascular bundle and several minor vascular bundles in normal leaves of the control, but there were several vascular bundles of more or less the same size in the trumpet-shaped leaves of treated ones. These results indicated that auxin polar transport played an important role on bilateral symmetry of leaf growth.
\end{abstract}

Key words: Brassica chinensis, Orychophragmus violaceus, Nicotiana tabacum, auxin polar transport, foliar modification. 
Gene delivery system targeting VEGF receptors

\section{INTRODUCTION}

Auxin polar transport plays an important role in different physiological processes of plants, such as apical dominance, cell division and differentiation,stem elongation and tropism[1],[2]. And it was demonstrated that auxin polar transport was essential for the establishment of bilateral symmetry during the development of zygotic embryos[3]. In particular, some reports suggested that disruption of auxin polar transport resulted in aberrant organ formation at the shoot apex[4-11]. When the tobacco leaf and pedicle explants were cultured on a medium containing auxin polar transport inhibitors, the formation of trumpet-shaped leaf-like organs was observed[4],[5]. In this paper, the systems of in vitro adventitious bud formation in the culture of Orychophragmus violaceus pedicel explants and Nicotiana tabacum leaf explants, and seedlings of Nicotiana tabacum and Brassica chinensis were used to show that diminution of auxin polar transport could induce the formation of shoot with trumpet-shaped leaves and/or fused leaves.

\section{MATERIAL AND METHODS}

\section{Auxin polar transport inhibitors}

Auxin polar transport inhibitors used in this study were 9-hydroxy-fluOrene-9-carboxylic acid (HFCA); 2, 3, 5-triiodobenzoic acid (TIBA) and trans-cinnamic acid (CA) (all from Sigma).

\section{Plant materials and culture}

The pedicels of Orychophragmus violaceus were surface-sterilized with saturated suspension of bleaching powder for $10 \mathrm{~min}$, washed with sterilized water for 3 times, and then cut into $1.5 \mathrm{~cm}$ long segment and placed on MS medium[12] containing zeatin (1 mg/L), IAA (1 mg/L) and different concentrations of the auxin polar transport inhibitor HFCA. The leaves of tobacco (Nicotiana tabacum L.) cv. Ge-xin No.1 were also surface-sterilized with the same method, and then cut into slices of $1 \times 1 \mathrm{~cm}_{2}$ and placed on MS medium containing 6-BA (2 mg/L) and different concentrations of any of the auxin polar transport inhibitors HFCA, TIBA and CA. The pedicel and leaf explants were checked and recorded after 30 days of culture. After surface-sterilization, seeds of tobacco and Brassica chinensis (cv.Ji-mao-cai) were germinated on MS medium containing an auxin polar transport inhibitor (HFCA or TIBA). The seedlings were checked and recorded after 60 days of culture. All the experiments were repeated more than 3 times.

\section{Histological observation}

The trumpet-shaped and fused leaves of $60 \mathrm{~d}$ cultured tobacco seedlings in the media containing auxin transport inhibitors and the normal leaves of tobacco seedlings in the control media were fixed with FAA solution for $24 \mathrm{~h}$, then the specimens were dehydrated, infiltrated, embedded and sectioned to a thickness of $10 \mu \mathrm{m}$. The sections were stained with hematoxylin and safranin by the routine method[13].

\section{RESULTS}

Effects of auxin polar transport inhibitors on adventitious bud formation from cultured explants

When the tobacco leaf explants were cultured on MS medium containing 6-BA 
Li JM et al.

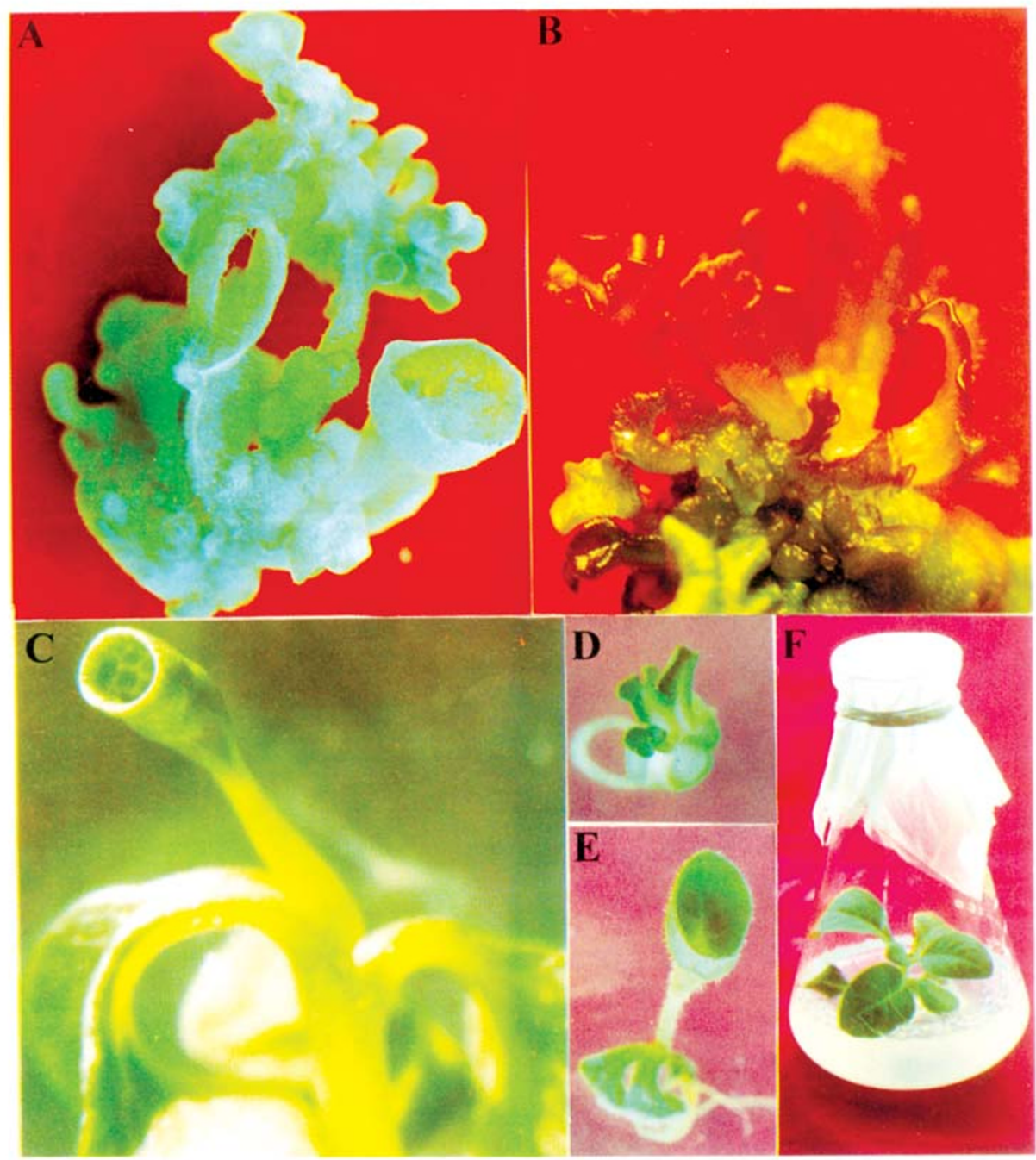

Fig 1. Trumpet-shaped leaves formed from cultured explants and seedlings on MS medium con taining auxin polar transport inhibitors; (A) Trumpet-shaped leaf formed from cultured tobacco leaf explants on MS medium containing HFCA and BA; (B) Trumpet-shaped leaf formed from cultured Orychophragmus violaceus pedicel explants on MS medium containing HFCA, zeatin and IAA; (C) Brassica chinensis seedling with trumpet-shaped leaf cultured on TIBA containing medium; (D) Brassica chinensis seedling with a fused leaf cultured on TIBA containing medium; (E) Tobacco seedling with a trumpet-shaped leaf cultured on TIBA containing medium;(F) Tobaacco seedling with normal leaves cultured on MS medium without inhibitors of auxiu polar transport $(\mathrm{CK})$. 
Gene delivery system targeting VEGF receptors

$(2 \mu \mathrm{g} / \mathrm{L})$ and the pedicel explants of Orychophragmus violaceus were cultured on MS medium containing zeatin $(1 \mu \mathrm{g} / \mathrm{L})$ and IAA $(1 \mu \mathrm{g} / \mathrm{L})$ for $30 \mathrm{~d}$, adventitious shoots were differentiated with normal leaf. But when auxin polar transport inhibitors were added into the above-mentioned medium, adventitious shoots were differentiated to trumpetshaped leaves and/or fused leaves (Fig 1A, B). The frequency of formation of adventitious shoots with trumpet-shaped leaves varied with plant species and the concentrations of the inhibitors used (Tab 1). Trumpet-shaped leaves and fused leaves could be formed in all the node positions of adventitious shoots in tobacco.

Tab 1. Effects of auxin polar transport inhibitor(s) at different concentrations on frequencies of adventitious shoot with trumpet-shaped leaves in the culture of Orychophragmus violaceus pedicel explants and Nicotiana tabacum leaf explants.

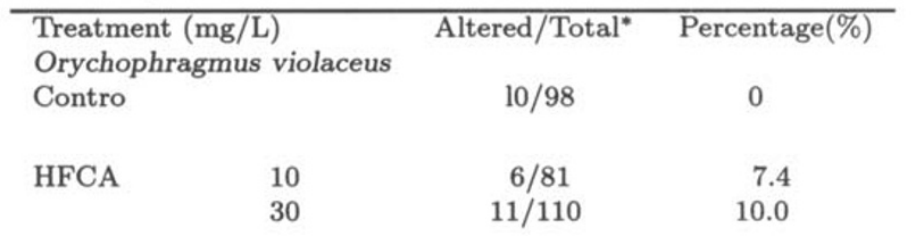

Nicotiana tabacum

Control

$0 / 1273 \quad 0$

TIBA

1
4
7.5

$13 / 425$

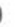

HFCA

$\begin{array}{ccc}2 & 35 / 738 & 4.74 \\ 7.5 & 46 / 584 & 7.88 \\ 20 & 56 / 769 & 7.28\end{array}$

\begin{tabular}{rrrr}
$\mathrm{CA}$ & 10 & $11 / 612$ & 1.80 \\
& 20 & $13 / 553$ & 2.35 \\
40 & $17 / 824$ & 2.06 \\
60 & $16 / 552$ & 2.90 \\
& 80 & $43 / 986$ & 4.36 \\
100 & $53 / 633$ & 8.37 \\
\hline
\end{tabular}

* Number of adventitious shoots with trumpet-shaped leaves/total number of adventitious shoots examined.

Effects of auxin polar transport inhibitors on leaf pattern formation of the seedlings

One of two auxin polar transport inhibitors (HFCA and TIBA) was added at various concentrations to the media in which the seeds were germinated. All the inhibitors used could induce the formation of trumpet-shaped and/or fused leaves (Fig 1C, D, 


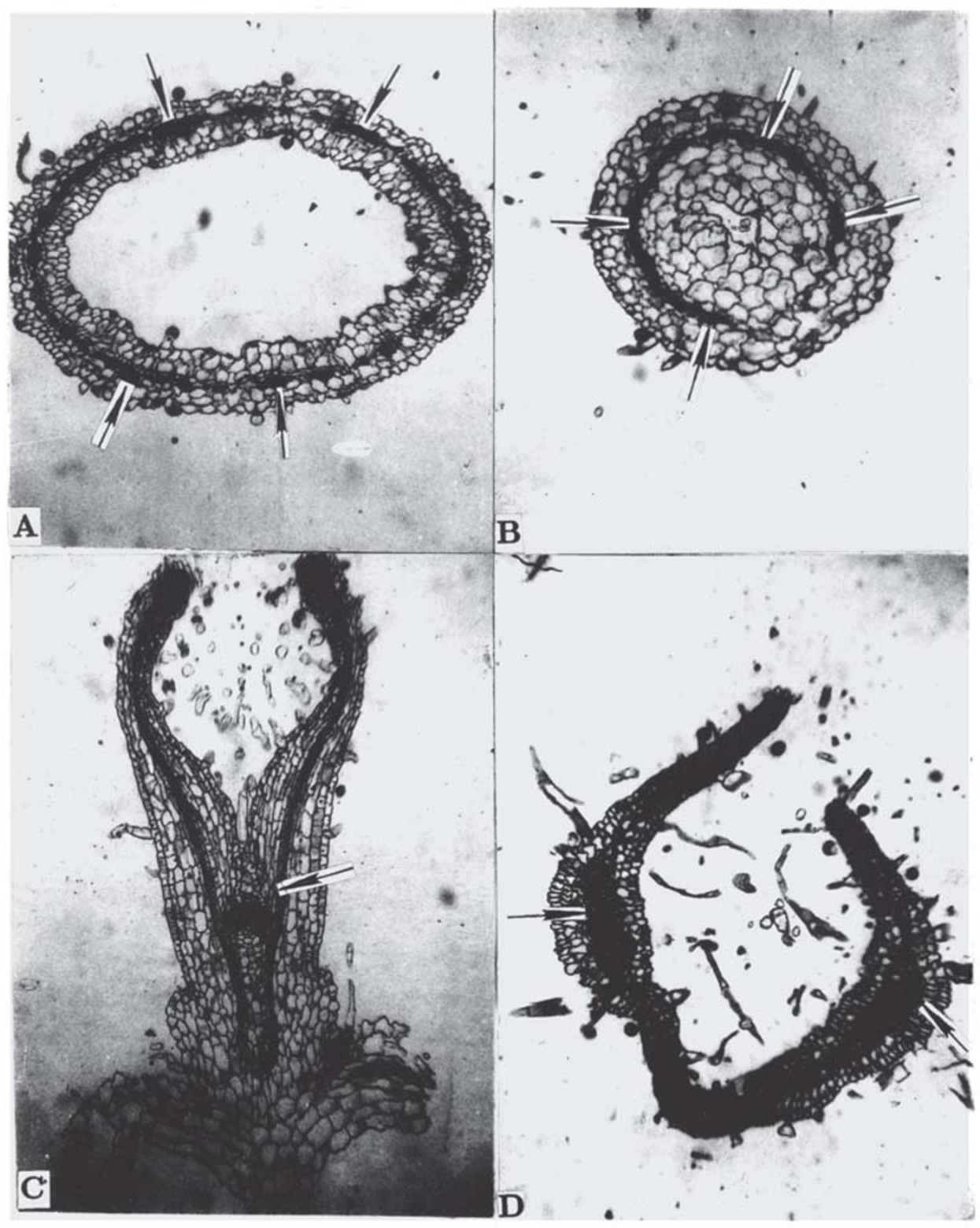

Fig 2. Transverse and longitudinal sections of tobacco seedlings with trumpet-shaped and/ or fused leaves (A) and (B) Transverse sections of seedlings with trumpet-shaped leaf: A-up part, B-lower part; showing forming a number of vascular bundles (arrow) of more or less the same size, instead of a normal big one (C) Longitudinal section of seedling with trumpet-shaped leaf, showing apical meristem (arrow) present at the basal part of the leaf (D) Transverse sections of seedling with fused leaf, showing two main vascular bundles (arrow) and some small ones present in the fused leaf. 
Gene delivery system targeting VEGF receptors

E). The frequency is related to concentrations of the inhibitors (Tab 2). Abnormal leaves were usually formed at the 4th or 5th leaf position of the seedlings.

The trumpet-shaped leaf could also be formed occasionally at the first foliage leaf position. In addition, HFCA and TIBA also inhibited root growth and induced formation of calli from roots of Brassica chinensis seedlings (e.g. $42.3 \%$ germinated on MS medium containing TIBA $15 \mu \mathrm{g} / \mathrm{L}$ ).

When the seedlings with trumpet-shaped leaves were transferred to a medium containing $1 / 2 \mathrm{MS}$ salts[12] for two months, normal shoots developed from the center of the trumpet-shaped leaves in some seedlings, but abnormal ones swelled at their base and could not grow normally.

\begin{tabular}{|c|c|c|c|}
\hline \multicolumn{2}{|c|}{ Treatment $(\mathrm{mg} / \mathrm{L})$} & Altered/Total ${ }^{*}$ & Percentage(\%) \\
\hline \multicolumn{4}{|c|}{ Brassica chinensis } \\
\hline \multicolumn{2}{|l|}{ Control } & $0 / 82$ & 0 \\
\hline \multirow[t]{2}{*}{ TIBA } & 15 & $3 / 59$ & 5.1 \\
\hline & 30 & $15 / 73$ & 20.5 \\
\hline \multirow[t]{2}{*}{ HFCA } & 15 & $13 / 51$ & 25.5 \\
\hline & 30 & $12 / 66$ & 18.2 \\
\hline \multicolumn{4}{|c|}{ Nicotiana tabacum } \\
\hline control & & $0 / 103$ & 0 \\
\hline \multirow[t]{3}{*}{ TIBA } & 7.5 & $4 / 104$ & 3.8 \\
\hline & 15 & $16 / 196$ & 8.2 \\
\hline & 30 & $12 / 117$ & 10.3 \\
\hline \multirow{3}{*}{ HFCA } & 7.5 & $8 / 75$ & 10.7 \\
\hline & 15 & $11 / 101$ & 10.9 \\
\hline & 30 & $10 / 74$ & 13.5 \\
\hline
\end{tabular}

* Number of plants with trumpet-shaped leaf/total number of plants examined

\section{Histological observation}

Histological examination was carried out to compare the trumpet-shaped and fused leaves with normal ones. In transverse section, there was only one main vascular bundle and several minor ones in normal leaves, but there were two main vascular bundles in those of fused leaves (Fig 2D) and more vascular bundles of more or less the same size in the trumpet-shaped leaves (Fig 2A, B). In longitudinal sections, there was meristem present at the basal part of trumpet-shaped leaves(Fig 2C).

\section{DISCUSSION}

Since the discovery of the polarity of auxin transport by Went[14] and its quantitative description by Van der Weij[15, 16], two questions have intrigued plant physiologists: 
Li JM et al.

how is the polarity of auxin transport achieved in plants, and what is its significance? Much knowledge about the effects of auxin polar transport on plant growth and development has been obtained by using the inhibitors and plant mutants of auxin polar transport[1,6].

In the previous work, it has been demonstrated that cylindrical cotyledons could be formed in Arabidopsis mutant (pin1-1)[3] and tobacco mutant (lat)[17] whose auxin polar transport activity was reduced. And it was reported that both the seedlings of wild type tobacco from the seeds germinated on the medium containing both cytokinin and inhibitors of auxin polar transport and the seedlings of ipttransgenic tobacco (whose cytokinin level has been elevated) on the medium containing auxin polar transport inhibitors alone developed saucer-shaped leaves, but the wild-type tobacco seedlings planted on medium containing the inhibitors alone did not develop that kind of abnormal leaflike organ[10]. However, the results presented here showed that inhibition of auxin polar transport by the inhibitors alone during seed germination of tobacco and Brassica chinensis was suffcient for the formation of trumpet-shaped leaves, possibly due to longer treatment in experiments. Inhibition of auxin polar transport could result in the formation of trumpet-shaped leaves in the cultured leaf explants of Crassula arboresiens and tobacco[4] and pedicel explants of Orychophragmus violaceus. And several reports have shown that disruption of auxin polar transport led to form cylindrical leaf-like organ at the shoot apex of Bryophyllum daigremontianum[7], Lens esculenta[11] and caused the formation of enlarged globular or oblong embryo in carrot somatic embryogenesis[9]. All these indicated that auxin polar transport is essential in foliage leaf pattern formation and supported the idea that cotyledons and foliage leaves are homologous structures[18].

Liu et al found that inhibitors of auxin polar transport could induce the formation of cylindrical cotyledons in Brassica juncea[3]. They proposed two possible models to explain the mechanism for forming cylindrical cotyledons. In both models, they proposed that auxin polar transport resulted in suitable concentration of auxin in cotyledon-forming regions to initiate the formation of cotyledon primordia. When its transport was blocked, the excess of auxin accumulated would be distributed through diffusion in a circular area so that cylindrical cotyledons were formed. Because of the similarity between the two phenomena (cylindrical cotyledones and the trumpet-shaped leaves described here). It is reasonable to believe that these models can also explain how the trumpet-shaped leaves were formed. In addition, it was reported that auxin transport inhibitors blocked the transport at the effux carrier[19]. But higher concentrations of auxin polar transport inhibitors were essential for blocking auxin transport. For example, CA (an inhibitor of auxin polar transport) at $100 \mathrm{mg} / \mathrm{L}$ could enhance significantly the frequency of trumpet-shaped leaf formation (Tab 1 ). The results presented here also showed that in most cases, trumpet-shaped leaf was formed at the 4 th $\sim 5$ th leaf node of the seedling, though some of them were formed at the different node including that of the first foliage leaf. The shape of leaf primordium has been considered as one of the three factors determining the final shape of leaf[20], thus it is possible that inhibition of auxin 
Gene delivery system targeting VEGF receptors

polar transport induced a change in the shape of leaf primordium to form trumpet-shaped leaves. Once normal leaf primordium has been formed, it seems not possible to develop such abnormal leaves. That could explain the phenomenon that trumpet-shaped or fused leaves were usually formed at 4 th or 5 th leaf node in our experiments. It was reported that making a shallow cut between the shoot apex and a leaf primordium when the latter was less than $50 \mu \mathrm{m}$ in length could make it grow into a taper-like one[21]. It implies that the signal exchange between primordium and shoot apex is important in the development of leaf primordium. Therefore, auxin polar transport inhibitors might interfere with this kind of signal transport by affecting the transport and distribution of auxin, and as a result, causes change in the shape of leaf primordium.

Okada et al found that in Arabidopsis mutant pin1 the auxin polar transport was inhibited and the phenotype of the mutant was the same as that of wildtype plants treated with auxin polar transport inhibitors[6]. This work correlates the mutants (the genes) and the auxin polar transport for the first time. Recently several pin genes have been cloned by Dr. Palme' s group in Max-Planck Institute of Plant Breeding in Köln, and one of them (pin1) is correlated to the mutant pin1-1 described by Okada. Due to the PIN protein predicted from pin1 sequence having typical membrane domain, they consider that PIN1 may be an auxin transporter[22]. According to the fact that protein kinase inhibitors could reduce the auxin polar transport activity in etiolated pea seedlings, it was suggested that inhibition of protein phosphorylation could lead to the hindrance of the auxin-exporting function of NPA receptors[23]. The research on pin gene, and its expression and regulation in plants will be helpful in understanding the effects of auxin polar transport on plant growth and development and their molecular mechanisms.

In conclusion, the data presented here showed that auxin polar transport plays an important role in leaf morphogenesis. Interference with this transport causes a failure in the formation of bilateral symmetry in foliage leaves and results in the formation of trumpet-shaped and/or fused leaves.

\section{ACKNOWLEGEMENT}

The authores want to thank Professor Ke Ming CUI (Life Sciences College, Peking University) for his technical help in histological observation.

This work was supported by Chinese National Natural Science Foundation (No. 39670078) and National Laboratory of Plant Molecular Genetics, Shanghai Institute of Plant Physiology, Chinese Academy of Sciences. 
Li JM et al.

\section{REFERENCES}

[1] Goldsmith MHM. The polar transport of auxin. Ann Rev Plant Physiol 1977; 28:439-78.

[2] Smulders MJM., Croes AF and Wullems GJ. Polar transport of 1-naphthaleneace acid determines the distribution of flower buds on explants of tobacco. Plant Physiology 1988; 88:752-6.

[3] Liu Chun-Ming, Xu Zhi-Hong and Chua Nam-Hai. Auxin polar transport is essential for the establishment of bilateral symmetry during early plant embryogenesis. Plant Cell 1993; 5:621-30.

[4] Ni Di-An, Wang Ling-Jian, Chen Yong-Ning, Xu Zhi-Hong and Xia Zhen-Ao. Inhibition of auxin polar transport affecting the model of growth and development. Acta Bot Sin 1996; 38:867-9.

[5] Chen Yong-Ning, Ni Di-An, Wang Ling-Jian, Li Wen-An, Xu Zhi-Hong. The Effects of TIBA on the floral bud differentiation from tobacco pedicels in vitro. Acta Biol Exp Sin 1997; 30:45-51.

[6] Okada K, Ueda J, Komaki MK, Bell CJ, Shimura Y. Requirement of the auxin transport system in early stage of Arabidopsis floral bud formation. Plant Cell 1991; 3:677-84.

[7] Ahmad SY. Morphactin-induced foliar modifications in Bryophllum daigremontianum. Phytomorphomology 1984; 34:81-3

[8] Meicenhrier RD. Changes in Epilobium phyllotaxy induced by N-1-naphthylphthaalamic acid and a-4-chlorophenoxyisobutyric acid. Amer J Bot 1981; 68:1139-54.

[9] Schiavone FM, Cooke TJ. Unusual patterns of somatic embrogenesis in the domesticated carrot: development effects of exogenous auxins and auxin transport inhibitors. Cell Diff 1987; 21:53-62.

[10] Strabala TJ, Wu Yan H, Li Yi. Combined effects of the auxin transport inhibitors and cytokinin: alterations of organ development in tobacco. Plant Cell Physiol 1996; 37:1177-82.

[11] Pillai VNK, Chakravati SC. Effect of certain synthetic growth regurators on form of leaf of Brassica campestris Linn and Lens esculenta Moench. Agra Univ J Res 1987; 3:317-22 227-230

[12] Murashige T, Skoog F. A rapid growth and bioassays with tobacco tissue cultures. Physiol Plant 1962; 15:473-97.

[13] Cui ke-ming, Wang Zhen. Hematoxylin-safranin staining of non-deparaffnated sections suitable for studying cambial activity. Acta Boreal Occident Sin 1997; 17:264-8.

[14] Went FW. Wuchsstoff und Wachstum. Rec Trav Bot Neerl 1928; 25:1-116.

[15] Weij Van der, Der HG. Mechanismus des Wuchsstoff transportes. Rec Trav Bot Neerl 1932; 29: 380-496.

[16] Weij Van der, Der HG. Mechanismus des Wuchsstoff transportes II. Rec Trav Bot Neerl 1934; 31:810-57.

[17] Naderi M, Caplan A, Berger PH. Phenotypic characterization of a tobacco mutant impaired in auxin polar transport. Plant Cell Reports 1997; 17:32-8.

[18] Kaplan DR, Cooke TJ. Fundamental concepts in the embrygenesis of dicotyledons:a morphological interpretation of embryo mutants. Plant Cell 1997; 9:1903-9.

[19] Jones AM. Auxin transport: down and out and up again. Science 1998; 282:2201-2.

[20] Esau K. The leaf. In: Katherine Esau (edi.) Plant Anatomy, 2nd Edition. pp.411-70.

[21] Sussex IM. Morphogenesis in Solanum tuberosum L.: Apical structure and developmental pattern of juvenile shoot. Phytomorphology 1955; 5:286-300.

[22] Galweiler L, Guan Chang-Hui, Muller A, Wisman E, Mendgen K, Yephremov A, Palme K. Regulation of polar auxin transport by AtPIN1 in Arabidopsis vascular tissue. Sciences 1998; 282:2226-30.

[23] Nam MH, Kang BG. Impairment of polar auxin transport by protein kinase inhibitors in etiolated pea seedlings. J Plant Biol 1995; 38:343-8. 